\title{
A Tensor-based Localization Framework Exploiting Phase Interferometry Measurements
}

\author{
Farzam Hejazi* \\ School of Electrical and \\ Computer Engineering \\ University of Central \\ Florida, Orlando \\ Email: farzam.hejazikookamari@ucf.edu
}

\author{
Mohsen Joneidi* \\ School of Electrical and \\ Computer Engineering \\ University of Central \\ Florida, Orlando \\ Email: joneidi@eecs.ucf.edu
}

\author{
Nazanin Rahnavard \\ School of Electrical and \\ Computer Engineering \\ University of Central \\ Florida, Orlando \\ Email: nazanin.rahnavard@ucf.edu
}

\begin{abstract}
In this paper, a framework for localization of multiple co-channel transmitters using phase difference measurements between two antennas mounted on sensors of a sensor network is proposed. To pursue localization, we equip each sensor with two antennas and we use temporal cross-correlations between the received signals of the two antennas to extract the phase differences between each antenna pairs, named as phase interferometry measurements (PIMs), provoked by each transmitters using tensor decomposition. We calculate Cramer-Rao lower bound of error of localization using PIMs. Our simulation results show that highly accurate estimations can be achieved using PIMs. We also compare the accuracy of our proposed technique with a sensor network that exploits highly directional linear array antennas and show that our proposed technique can perform similar to a network that employs very large antenna arrays.
\end{abstract}

\section{INTRODUCTION}

Separating power spectra and localization of unrevealed sources have several applications in electronic warfare, mobile wireless networks and cognitive radio networks to just name a few. This type of electromagnetic environmental awareness is necessary for electronic support (ES) systems to detect adversarial radio activities and localize hostile transmitters [1]. In cognitive radio and mobile networks, localization of primary users (PUs) and mapping their power spectra, referred to as radio cartography, are crucial for spatial frequency reuse and spectrum management [2]. Bazerque. et. al. suggest using a network of omnidirectional antennas to measure signal strength at different locations around the network and introduce a technique for joint estimation of power spectral density (PSD) in space and frequency [3], with the assumption of a sparse placement of PUs in the network. In a more recent work, this technique is expanded by using a network of linear array antennas that exploit beamforming for tracking sources and dynamic radio cartography [4]. The requirement of having prior information on channel gain magnitudes between sensors and potential locations of PUs is the main deficiency of these techniques.

In the passive localization literature, various techniques have been proposed to pursue the localization task. A class of

\footnotetext{
* indicates shared first authorship. This material is based upon work supported by the National Science Foundation under Grant No. CCF-1718195.
}

techniques employs direction-of-arrival (DOA) or angle-ofarrival (AOA) to estimate target locations and track moving targets [5], [6]. Time-of-arrival (TOA) and time-differenceof-arrival (TDOA) of received signals in a sensor network are widely used in literature to find locations of targets [7]. Frequency difference of arrival (FDOA) in different sensors also can be employed to localize moving emitters in an environment [8]. Lately, methods that use a combination of two or three of AOA/TDOA/FDOA techniques have gained more attention [9], [10]. Phase interferometry is widely used in electronic warefere applications for wide-band (WB) interferometric direction finding [11], [12], [13]. Since phase difference between two antennas introduces ambiguous DOAs, most techniques proposed in the literature suggest using two or more baselines to disambiguate phase difference [14], [15]. Here, we suggest measuring phase differences using a sensor network that collects PIMs from different geographical locations to disambiguate the phase and directly translate phase differences to PUs locations.

Tensor decomposition is a strong mathematical tool for modeling and analysis of multi-dimensional data. Tensorbased methods have been employed in communications and coding frameworks since Sidiropoulos et. al. introduced them for blind code-division multiple access (CDMA) [16]. Recently, more advanced coding methods in communication systems are developed based on the tensor decomposition theory [17], [18]. In addition to coding, tensor decomposition has received attentions in vehicular communication [19], channel estimation [20] and data compression [21]. Moreover, tensorbased techniques are employed for localization-based spectrum sensing [22], [23]. These localization methods employ attenuation and phase shift from sources to antennas at receivers and each sensor is equipped with a single antenna. In the present work, we employ a tensor decomposition technique to extract phase differences in a set of paired antennas on each sensor of the network. The main contribution in this paper can be encapsulated as follow:

- Introducing a tensor-based approach to separate power spectra of PUs and to extract PIMs on each sensors provoked by each PU 
- Putting forward a localization scheme that employs PIMs

- Deriving Cramer-Rao lower bound (CRLB) of location estimation using PIMs.

Notations: Throughout this paper, vectors, matrices, and tensors are denoted by bold lowercase, bold uppercase, and bold underlined uppercase letters, respectively. If $\underline{\boldsymbol{W}} \in$ $\mathbb{R}^{N_{1} \times N_{2} \times N_{3}}$ then $(\underline{\boldsymbol{T}})_{:, n_{2}, n_{3}}$ is a vector of length $N_{1}$, also known as a mode-1 fiber of $\underline{W}$, defined by fixing all the indices but one. Similarly, we have mode-2 and mode- 3 fibers. $\boldsymbol{W}^{(1)}, \boldsymbol{W}^{(2)}$, and $\boldsymbol{W}^{(3)}$ are unfolded matrices whose columns are fibers of the first, second and third mode of $\underline{\boldsymbol{W}}$, respectively. Moreover, $\circ$ denotes the outer product, i.e., entries of $\underline{\boldsymbol{W}}=\boldsymbol{a} \circ \boldsymbol{b} \circ \boldsymbol{c}$ is calculated as $w_{n_{1} n_{2} n_{3}}=a\left(n_{1}\right) b\left(n_{2}\right) c\left(n_{3}\right)$ and vec(.) is an operator that concatenates columns of a matrix into a vector.

The rest of the paper is ordered as follows. Section II formulates the problem. Power spectra separation and PIM extraction from temporal cross-correlation matrix of received signals through a tensor decomposition approach are introduced in Section III. We analyze CRLB of localization using PIM in Section IV. Section V presents experimental results. Finally Section VI concludes the paper.

\section{Problem Statement AND Preliminaries}

We consider a scenario where there are $K$ transmitters (or PUs) in a region of interest. A sensor network including $N$ sensors co-exists with PUs and each sensor is equipped with two antennas whose distance is indicated by $D$ (see Figure 1). Distance between antennas of a ULA is at most half of the wavelength [24], however, in our proposed technique there is no constraint on $D$. Here, our main goal is to find locations of all PUs. We presume that the environment is multi-path free. Given these assumptions, received signals at two antennas of sensor $n$ can be simply formulated as

$$
\begin{aligned}
& u_{1 n}(t)=\sum_{k=1}^{K} a_{n, k} g_{k}\left(t-\Delta t_{n, k}^{(1)}\right)+v_{1 n}(t), \\
& u_{2 n}(t)=\sum_{k=1}^{K} a_{n, k} g_{k}\left(t-\Delta t_{n, k}^{(2)}\right)+v_{2 n}(t),
\end{aligned}
$$

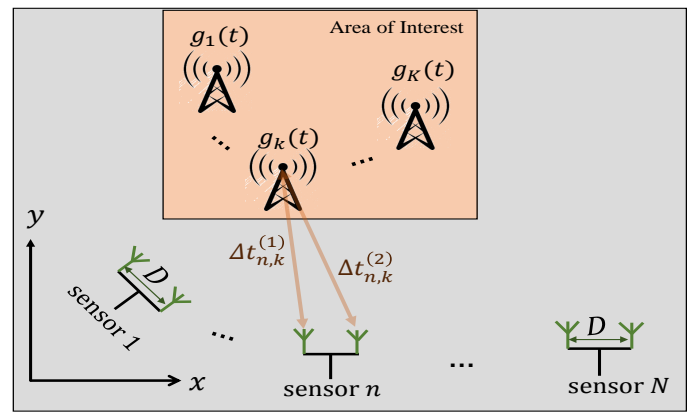

Fig. 1. The geometry of the localization problem. A sensor network utilizes sensors equipped with two antennas to measure phase differences between antenna pairs. where $a_{n, k}$ marks channel gain between the $n^{\text {th }}$ sensor and the $k^{t h} \mathrm{PU}, \Delta t_{n, k}^{(1)}$ and $\Delta t_{n, k}^{(2)}$ are the time delays of arrival of signal from PU $k$ to the first and the second antennas of sensor $n$, respectively, $v_{1 n}$ and $v_{2 n}$ mark white-Gaussian complex noise at each antennas of sensor $n, g_{k}(t)$ marks the transmitted signal of the $k^{t h} \mathrm{PU}$ as

$$
g_{k}(t)=m_{k}(t) e^{-j 2 \pi f_{c} t},
$$

where $m_{k}(t)$ is a base-band message, $g_{k}(t)$ is assumed to be narrow-band and wide-sense stationary, and $f_{c}$ is the carrier frequency. Here, we assume that transmitters are co-channel and therefore $f_{c}$ is the same for all PUs. We also assume that all $N$ sensors of the network are synchronized. The TDOA between two antennas at sensor $n$ can be reformulated as

$$
\Delta t_{n, k}^{(1)}-\Delta t_{n, k}^{(2)}=\Delta t_{n, k}=\frac{r_{k, n}^{(1)}-r_{k, n}^{(2)}}{c},
$$

where $r_{k, n}^{(1)}$ and $r_{k, n}^{(2)}$ denote the distances between the PU $k$ and first and seconds antennas of sensor $n$, respectively, and $c$ marks the speed of light. We have

$$
\begin{aligned}
& r_{n, k}^{(1)}=\sqrt{\left(x_{s_{n}}^{(1)}-x_{P U_{k}}\right)^{2}+\left(y_{s_{n}}^{(1)}-y_{P U_{k}}\right)^{2}} \\
& r_{n, k}^{(2)}=\sqrt{\left(x_{s_{n}}^{(2)}-x_{P U_{k}}\right)^{2}+\left(y_{s_{n}}^{(2)}-y_{P U_{k}}\right)^{2}},
\end{aligned}
$$

where $\left(x_{s_{n}}^{(1)}, y_{s_{n}}^{(1)}\right),\left(x_{s_{n}}^{(2)}, y_{s_{n}}^{(2)}\right),\left(x_{P U_{k}}, y_{P U_{k}}\right)$ are locations of the first and the second antennas of sensor $n$ and the $k^{\text {th }}$ $\mathrm{PU}$, respectively. Given base-band messages are narrow band, $u_{1 n}(t)$ and $u_{2 n}(t)$ can be formulated as [25]

$$
\begin{aligned}
& u_{1 n}(t)=\sum_{k=1}^{K} a_{n, k} g_{k}(t)+v_{1 n}(t) \\
& u_{2 n}(t)=\sum_{k=1}^{K} a_{n, k} e^{-j 2 \pi \frac{1}{\lambda}\left(r_{n, k}^{(1)}-r_{n, k}^{(2)}\right)} g_{k}(t)+v_{2 n}(t) .
\end{aligned}
$$

We form $\boldsymbol{u}_{1}(t)=\left[u_{11}(t), \ldots, u_{1 N}(t)\right]^{T}$ and $\boldsymbol{u}_{2}(t)=$ $\left[u_{21}(t), \ldots, u_{2 N}(t)\right]^{T}$ by integrating received signals from the first and the second antennas of each sensors in two vectors. In the next section we show how to collect information of all sensors in a three-dimensional tensor and measure PIMs. Tensor decomposition is exploited to perform a collaborative localization in order to localize all targets simultaneously.

Our proposed tensor-based approach is mainly based on CANDECOMP/PARAFAC (CP) decomposition [26], which factorizes a tensor into a sum of rank-one tensors. $\mathrm{CP}$ decomposition is an extension of singular value decomposition (SVD) which facilitates a wide range of applications in sensor networks [27]. For example, a three-way tensor $\underline{\boldsymbol{W}} \in \mathbb{R}^{N \times N \times N_{F}}$ of rank $K$ can be decomposed as

$$
\underline{\boldsymbol{W}}=\sum_{k=1}^{K} \boldsymbol{a}_{k} \circ \boldsymbol{b}_{k} \circ \boldsymbol{c}_{k}=\llbracket \boldsymbol{A}, \boldsymbol{B}, \boldsymbol{C} \rrbracket,
$$

where $\boldsymbol{a}_{k} \in \mathbb{R}^{N}, \boldsymbol{b}_{k} \in \mathbb{R}^{N}$ and $\boldsymbol{c}_{k} \in \mathbb{R}^{N_{F}}$ are factor vectors of the $k^{\text {th }}$ rank-one component (Fig. 2). The factor matrices 
refer to the collection of factor vectors from the rank-one components, i.e., $\boldsymbol{A}=\left[\begin{array}{llll}\boldsymbol{a}_{1} & \boldsymbol{a}_{2} & \ldots & \boldsymbol{a}_{K}\end{array}\right]$ and likewise for $\boldsymbol{B}$ and $\boldsymbol{C}$. The kruskal-rank which is also referred to as krank is used to derive conditions on uniqueness of tensor decomposition for matrices $\boldsymbol{A}, \boldsymbol{B}$, and $\boldsymbol{C}$ [26]. krank is defined as the maximum number $k$, such that any $k$ columns are linearly independent [26].

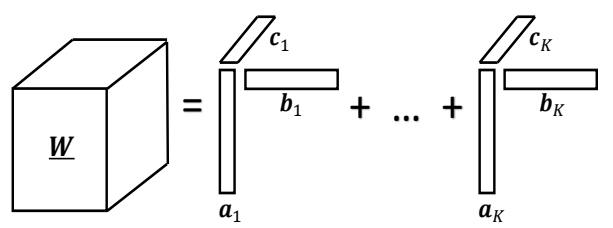

Fig. 2. Schematic of CP decomposition to summation of $K$ rank-one tensors. Each rank one tensor of dimension 3 can be written as outer product of 3 vectors.

\section{Phase InTERFEROMETRY LOCALIZATION USING TENSOR DECOMPOSITION}

In the previous section we modeled the received signal at each antenna pairs of sensors and showed that each PU provokes a specific phase difference on each sensor. We also showed that phase differences are merely functions of PUs' locations (which are unknown) and antennas' locations (which are known). In this section we are going to inaugurate a technique to uncover these phase differences from temporal cross-correlation of received signals. Let

$$
\boldsymbol{R}_{\boldsymbol{u}_{1} \boldsymbol{u}_{2}}(\tau)=\mathrm{E}\left\{\boldsymbol{u}_{1}(t) \boldsymbol{u}_{2}^{H}(t-\tau)\right\}
$$

represent cross-correlation between $\boldsymbol{u}_{1}$ and $\boldsymbol{u}_{2}$ at time lag $\tau$. We can reformulate $\boldsymbol{u}_{1}(t)$ and $\boldsymbol{u}_{2}(t)$ as

$$
\begin{aligned}
& \boldsymbol{u}_{1}(t)=\boldsymbol{A g}(t)+\boldsymbol{v}_{\mathbf{1}}(\boldsymbol{t}), \\
& \boldsymbol{u}_{2}(t)=\boldsymbol{B g}(t)+\boldsymbol{v}_{\mathbf{2}}(\boldsymbol{t}),
\end{aligned}
$$

where $\boldsymbol{g}(t)=\left[g_{1}(t), \ldots, g_{K}(t)\right]^{T}$ is the source signals of $K$ PUs, $\boldsymbol{v}_{1}(t)=\left[v_{11}(t), \ldots, v_{1 N}(t)\right]^{T}$ and $\boldsymbol{v}_{2}(t)=$ $\left[v_{21}(t), \ldots, v_{2 N}(t)\right]^{T}$ are received white Gaussian noise at the first and the second antennas of all sensors, respectively. Moreover, according to (7) and (8), $\boldsymbol{R}_{\boldsymbol{u}_{1} \boldsymbol{u}_{2}}$ can be reformulated as

$$
\boldsymbol{R}_{\boldsymbol{u}_{1} \boldsymbol{u}_{2}}(\tau)=\boldsymbol{A} \boldsymbol{D}(\tau) \boldsymbol{B}^{H}
$$

where $\boldsymbol{D}(\tau)=\operatorname{Diag}(\boldsymbol{\rho}(\tau)), \boldsymbol{\rho}(\tau)=\left[\rho_{1}(\tau), \ldots, \rho_{K}(\tau)\right]^{T}$, and $\rho_{k}(\tau)=\mathrm{E}\left\{g_{k}(t) g_{k}^{*}(t-\tau)\right\}$ marks auto-correlation function of $g_{k}(t)$ in time-lag $\tau$. Moreover, according to (5b), each element of matrix $\boldsymbol{B}=\left[b_{n, k}\right] \in \mathbb{R}^{N \times K}$ equals to the corresponding element in matrix $\boldsymbol{A}=\left[a_{n, k}\right] \in \mathbb{R}^{N \times K}$ multiplied by a complex phase difference, i.e.,

$$
b_{n, k}=a_{n, k} e^{-j 2 \pi \frac{1}{\lambda}\left(r_{k, n}^{(1)}-r_{k, n}^{(2)}\right)} .
$$

This phase difference within each pair of antenna plays the key role in our proposed localization method. We define $\Delta \phi=\left[\Delta \phi_{n, k}\right]$, where $\Delta \phi_{n, k}=\frac{b_{n, k}}{a_{n, k}}=e^{-j 2 \pi \frac{1}{\lambda}\left(r_{k, n}^{(1)}-r_{k, n}^{(2)}\right)}$, to represent phase difference between all elements of $\boldsymbol{A}$ and
$\boldsymbol{B}$. We refer to $\boldsymbol{\Delta} \boldsymbol{\phi}$ as PIMs. Let $\boldsymbol{z}(\tau)=\operatorname{vec}\left(\boldsymbol{R}_{\boldsymbol{u}_{1} \boldsymbol{u}_{2}}(\tau)\right)$, and $Z_{i}(w)$ be the discrete Fourier transform ${ }^{1}$ of the $i^{\text {th }}$ $\left(i=1, \ldots, N^{2}\right)$ element of $\boldsymbol{z}(\tau)[22]$

$$
Z_{i}(\omega)=\sum_{\tau=-\infty}^{\infty} z_{i}(\tau) e^{-j \omega \tau} .
$$

By defining $W_{i}(f)=Z_{i}\left(\frac{2 \pi f}{N_{F}}\right)$, for $f=0, \ldots, N_{F}-1$, we construct vectors $\boldsymbol{w}_{i}=\left[W_{i}(0), \cdots, W_{i}\left(N_{F}-1\right)\right]^{T}$ for $i=$ $1, \ldots, N^{2}$. Let $\boldsymbol{W}^{(3)}=\left[\boldsymbol{w}_{1}, \cdots, \boldsymbol{w}_{N^{2}}\right]$ which is the unfolded replica of tensor $\underline{W}$ w.r.t. the third dimension. The tensor representation can be expressed as

$$
\underline{\boldsymbol{W}}=\llbracket \boldsymbol{A}, \boldsymbol{B}^{*}, \boldsymbol{C} \rrbracket,
$$

where $\boldsymbol{C}=\left[\boldsymbol{c}_{1}, \ldots, \boldsymbol{c}_{K}\right]^{T}$ corresponds to propagating PSD from all PUs and entries of $\boldsymbol{c}_{k}=\left[S_{k}(0), \ldots, S_{k}\left(N_{F}-1\right)\right]^{T}$ are sampled from Fourier transform of the $k^{\text {th }}$ PU auto-correlation which is defined as follows

$$
S_{k}(w)=\sum_{\tau=-\infty}^{\infty} \rho_{k}(\tau) e^{-j w \tau} .
$$

The model in (12) is a PARAFAC model. Using a tensor rank decomposition technique, $\boldsymbol{A}, \boldsymbol{B}^{*}, \boldsymbol{C}$ can be recovered up to a scale and permutation in columns. There is a limit on the number of detectable PUs $(K)$ imposed by a constraint that guarantees identifiability of PARAFAC decomposition [28]

$$
\operatorname{krank}(\boldsymbol{A})+\operatorname{krank}(\boldsymbol{B})+\operatorname{krank}(\boldsymbol{C}) \geq 2 K+2,
$$

where $\operatorname{krank}(\boldsymbol{X})$ denotes the Kruskal rank of the matrix $\boldsymbol{X}$.

Remark 1. Given $\boldsymbol{A}, \boldsymbol{B}, \boldsymbol{C}$ are full rank and $N_{F} \geq K \geq 2$, the following upper bound holds over the number of detectable PUs

$$
K \leq 2 N-2 .
$$

proof. Assume $\boldsymbol{A}$ and $\boldsymbol{B}$ are $N \times K$ full rank matrices and $C$ is a $N_{F} \times K$ full rank matrix. For $K<N$, we have

$$
\operatorname{krank}(\boldsymbol{A})=\operatorname{krank}(\boldsymbol{B})=\operatorname{krank}(\boldsymbol{C})=K,
$$

and (13) is relaxed to $K \geq 2$ that is assumed. For $K \geq N$, we have

$$
\operatorname{krank}(\boldsymbol{A})=\operatorname{krank}(\boldsymbol{B})=N, \text { and } \operatorname{krank}(\boldsymbol{C})=K .
$$

Referring to (13), it concludes that $K \leq 2 N-2$.

Since $\boldsymbol{A}$ and $\boldsymbol{B}$ are channel gain matrices from PUs to sensors, it is very probable that $\boldsymbol{A}$ and $\boldsymbol{B}$ are full rank. Rank of $\boldsymbol{C}$ depends on PUs' signals spectral densities. In particular, if PUs emit signals that are uncorrelated in frequency spectrum domain, it is very probable that $\boldsymbol{C}$ is full rank. Intuitively, we can reason that the number of detectable PUs decreases if PUs' signals are correlated in spectrum domain or channel

\footnotetext{
${ }^{1}$ Cross-correlation $\boldsymbol{R}_{\boldsymbol{u}_{1}} \boldsymbol{u}_{2}(\tau)$ contains $N^{2}$ entries that each one is a function of $\tau$. Thus, corresponding to each entry a Fourier transform can be computed over variable $\tau$. We can use either Fourier transform or discrete Fourier transform. Here, we choose discrete Fourier transform since it is more applicable in practice.
} 
gian matrices are not full rank, since it would be much more difficult to discriminate between PUs.

Since all elements of $\boldsymbol{C}$ are non-negative, the phase difference between each element of $\boldsymbol{B}$ and $\boldsymbol{A}$ can be calculated. Consequently we can retrieve the matrix of phase differences $\Delta \phi$ up to a permutation in columns. Each column of $\Delta \phi$ shows phase differences provoked by one of the PUs in all sensors, and consequently it can be processed for localization. Let $\Delta \phi_{k}$ be the $k^{t h}$ column of $\Delta \phi$,

$$
\boldsymbol{\Delta} \phi_{k}=\left[e^{-j 2 \pi \frac{1}{\lambda}\left(r_{k, 1}^{(1)}-r_{k, 1}^{(2)}\right)}, \ldots, e^{-j 2 \pi \frac{1}{\lambda}\left(r_{k, N}^{(1)}-r_{k, N}^{(2)}\right)}\right]^{T}
$$

Given known positions of sensors, $\Delta \phi_{k}$ is only a function of unknown parameters $\left(x_{P U_{k}}, y_{P U_{k}}\right)$ which is the position of $k^{t h}$ PU. To recover the position of the corresponding PU, we solve the following optimization problem

$$
\left(x_{P U_{k}}, y_{P U_{k}}\right)=\underset{x, y}{\operatorname{argmin}}\left\|\boldsymbol{\Delta} \phi(x, y)-\Delta \phi_{k}\right\|_{2},
$$

where $\Delta \phi(x, y)$ is formulated the same as $\Delta \phi_{k}$ except that $\left(x_{P U_{k}}, y_{P U_{k}}\right)$ is replaced by $(x, y)$. In other words, $(15)$ is a simple two-dimensional search on all possible positions in the area of interest to find the location of the $k^{\text {th }}$ PU. In practice, we assume a grid with $N_{g}$ cells over the area of interest and perform the search on that grid. $N_{g}$ is a function of the size of the area of interest and the localization resolution. The whole process of localization using PIMs is summarized in Alg. 1 and Figure 3.
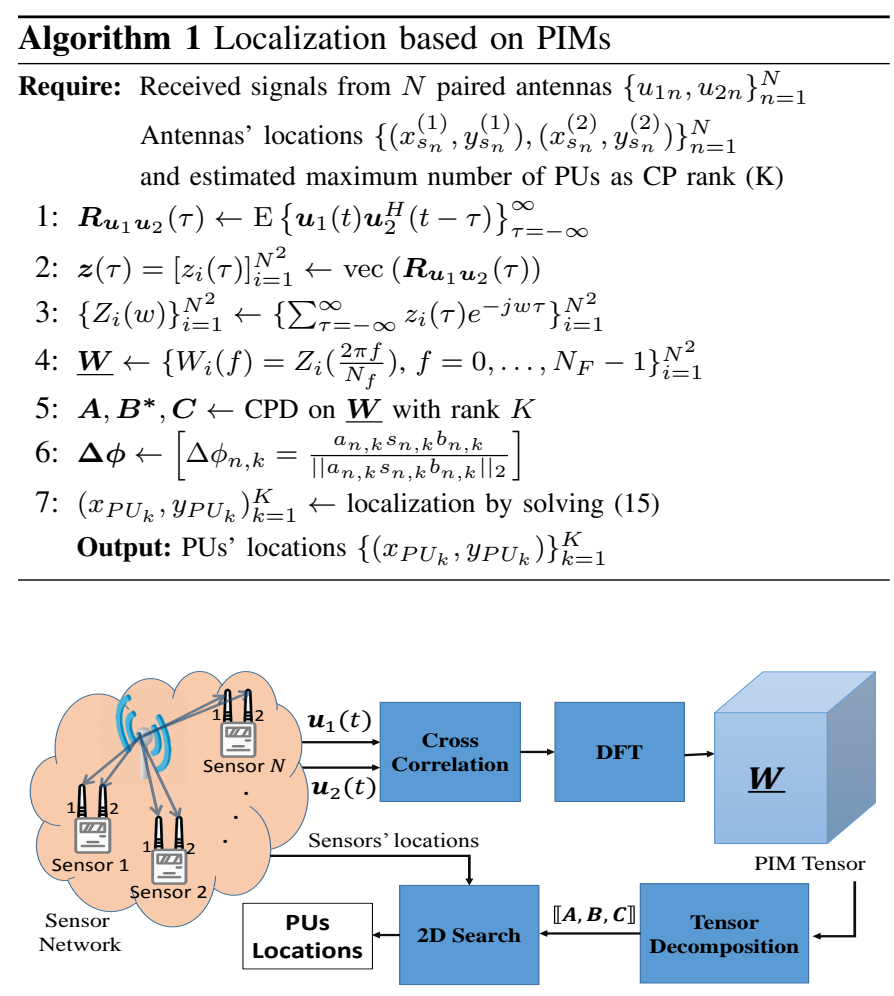

Fig. 3. The block diagram of the proposed localization scheme.
Considering Alg. 1, computational cost of step 2 is $O\left(N^{2} \log (N)\right)$, in case we approximate continuous Fourier transforms by FFT with length $N^{2}$ [29]. Assuming $N_{F} \geq$ $\max (N, K)$, the computational cost of step 5 is $O\left(K N_{F}^{3}\right)$ [30]. The computational cost of step 8 is $O\left(N N_{g}\right)$. Therefore, overall computational cost of the proposed algorithm can be determined either by step 5 or step 8 , depending on which one of them is greater.

\section{CRAmer-RaO Lower Bound of ERror (CRLB)}

In this section we investigate CRLB for an unbiased estimator that employs PIMs as of (14). As we mentioned in the previous section, we recovered phase differences between two antennas of each sensor provoked by a PU. Given each measurement is added by an independent white Gaussian noise, Fisher information matrix (FIM) of phase difference measurements from PU $k$ can be calculated as [31]

$$
\boldsymbol{J}\left(x_{P U_{k}}, y_{P U_{k}}\right)=\frac{1}{\sigma^{2}} \sum_{i=1}^{N} \nabla_{x, y}\left(\boldsymbol{\Delta} \phi_{k}\right)_{i}^{H} \nabla_{x, y}\left(\boldsymbol{\Delta} \phi_{k}\right)_{i},
$$

where $\sigma^{2}$ is the variance of noise, $\left(\Delta \phi_{k}\right)_{i}$ is the element $i$ of $\boldsymbol{\Delta} \phi_{k}$ and $\nabla_{x, y}$ is gradient operator w.r.t $x, y$. Consequently CRLB of variance of error turns into

$$
\begin{aligned}
\mathrm{E}\left\{\left(x_{P U_{k}}-\bar{x}_{P U_{k}}\right)^{2}+\right. & \left.\left(y_{P U_{k}}-\bar{y}_{P U_{k}}\right)^{2}\right\} \geq \\
& \operatorname{trace}\left(\boldsymbol{J}\left(x_{P U_{k}}, y_{P U_{k}}\right)^{-1}\right),
\end{aligned}
$$

where $\left(\bar{x}_{P U_{k}}, \bar{y}_{P U_{k}}\right)$ is the estimated position of PU $k$. To examine, the lower bound of error that can be achieved by employing measurements in the form of (14), we conduct several simulations. As an example, assume that 9 sensors are placed in a line align with the left edge of the area of interest. CRLB inside the area of interest is depicted in Fig. 4. As the figure illustrates CRLB varies from sub-meter values near the edge closest to the sensor network, to more than two meters near the two farthermost vertices to the sensor network. Although in this simulation we suppose that signal-to-noiseratio $(S N R)$ is $20 \mathrm{~dB}$, much higher SNRs is accessible due to the independency of noise in antennas pairs that results in the cancellation of noise terms in (9).

Assuming identical SNR at input antennas, CRLB illustrated in Figure 4 is very close in shape and values to CRLB of the same sensor network that utilizes directional antennas with $0.02^{\circ}$ in standard deviation of directional of arrival (DOA) estimation error [31], [32]. Such a precision in DOA estimation can be achieved using a ULA with at least 80 antennas when $S N R=20 d B$ [33].

\section{Simulation Results}

As we discussed in Section III, to localize multiple PUs in an area of interest, we obtain PIMs for each PUs using a PARAFAC tensor decomposition. To represent the cost function of localization when measurements are not noisy, let us define

$$
f(x, y)=\left\|\boldsymbol{\Delta} \phi(x, y)-\boldsymbol{\Delta} \phi_{x_{0}, y_{0}}\right\|_{2},
$$




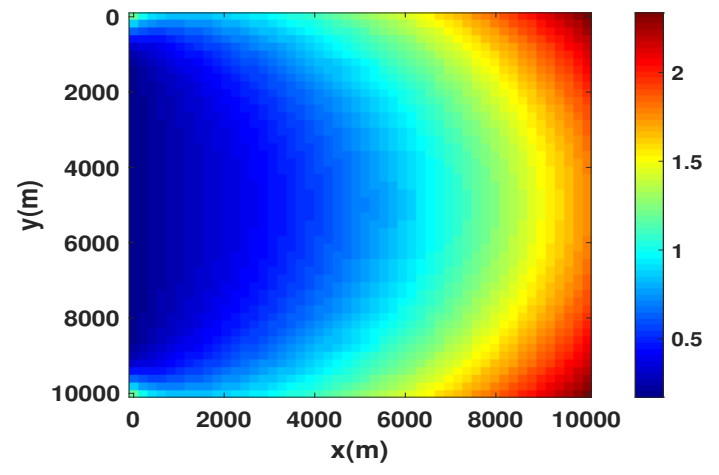

Fig. 4. Standard deviation of CRLB of location estimation using PIMs of (14). In this scenario, 9 sensors are placed in a line aligned $y$ axis, the first one is placed at $(1,-1) K m$, the ninth one is placed at $(9,-1) K m$, and all other sensors are placed in equi-spaced locations on a line that connects them. Two antennas of each sensors are placed $10 \mathrm{~m}$ apart aligned with $y$-axis , $D=10 \mathrm{~m}, f_{c}=5 \mathrm{GHz}$, and $S N R=20 d B$.

where $\Delta \phi_{x_{0}, y_{0}}$ denotes PIMs of an arbitrary known PU placed at $\left(x_{0}, y_{0}\right)$. In our first simulation, we plot $f(x, y)$ for a PU that is placed at $(500,500) m$, sensors are placed in a line aligned $y$ axis from $(1,-1) K m$ to $(9,-1) K m$ in equi-spaced locations, in particular, the first sensor is placed at $(1,-1) K m$ and the ninth sensor placed at $(9,-1) K m$, all the remaining sensors placed between these two sensors. D is set to $10 \mathrm{~m}$. As Figure 5 illustrates, $f(x, y)$ is a non-convex function that possesses several local minimum and maximum; however, there is a global minimum at the location of PU that can be revealed by search.

In the following simulation, we compare the accuracy of localization between two following sensor network. The first sensor network is equipped with two omni-directional antennas at each sensors which measures PIMs and localizes PUs using the proposed algorithm, while the second sensor network is equipped with ULAs, each uses $M$ antennas, and exploits DOA localization techniques. Sensor placements of both networks are the same. We suppose that power of received signals at each antennas of sensor $n$ of the first and the second sensor networks are identical, which means input SNRs of all antennas in both scenarios are the same. However, since each array of the second network, receives signal at $M$ antennas,

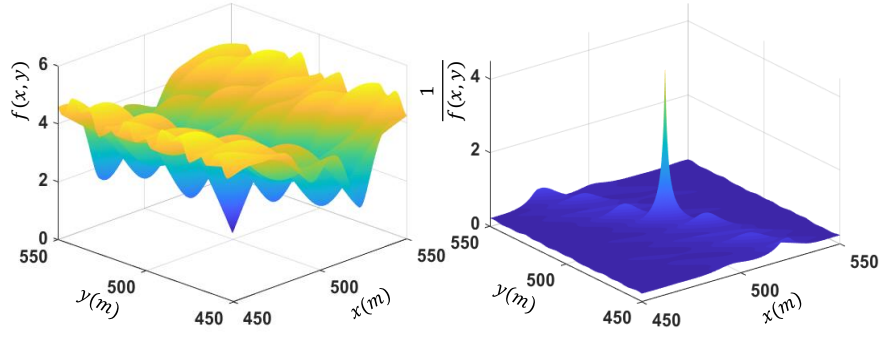

Fig. 5. (a) Cost function of localization $(f(x, y))$ for a PU located at $(500,500) m,(b)$ For a better visualization the reciprocal of the same function $\left(\frac{1}{f(x, y)}\right)$ is depicted. In this simulation, the scenario is the same as of Figure 4.

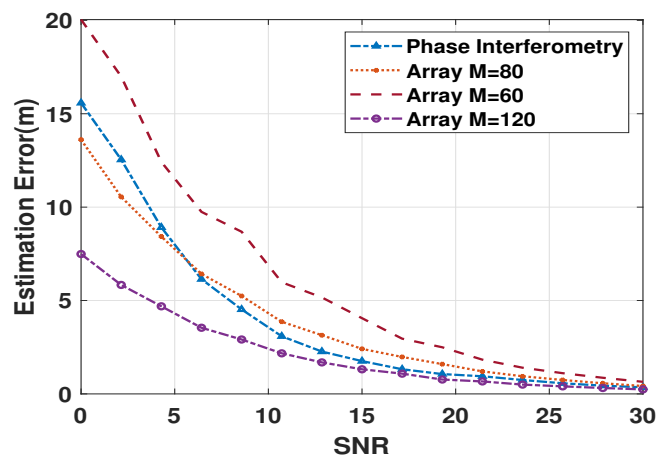

Fig. 6. Comparison of location estimation errors (as definition of (17)) between a sensor network that uses 2 antennas mounted on each sensors and phase interferometry measurements and a sensor network that uses ULAs with $M$ antennas mounted on each sensor. The scenario is the same as of Figure 4.

it can integrate all $M$ signals to obtain a better SNR, while each array of the first network receives signals at only two antennas and can't take advantage of the array gain in the first place. Nonetheless, referring to (7), the first network can make use of independency of noise at first and second antennas to mitigate noise level heavily. Given only one PU is located in the area, we assume that each antenna of the second network measures DOA of the signal transmitted by the PU utilizing spatial search at each array [34], and consequently localizes the PU using the total least square technique [35]. We also assume that antennas of each ULA are placed half wave-length apart.

Figure 6 illustrates that the accuracy of the proposed technique that only mounts 2 antennas with 166 wavelengh distance apart on each sensor is in the vicinity of a network that mounts arrays with $M=80$ elements on each sensor when SNR is between $3 \mathrm{~dB}$ to $7 \mathrm{~dB}$, and is better than the same array network when SNR is above $7 \mathrm{~dB}$. This leads to a huge reduction in complexity of sensor's antenna, since ULAs should be equipped with phase shifters behind each antennas and a complex receiver for beam steering [36]. Conversely, in the proposed schema the phase difference between only two antennas is measured. Moreover, antenna arrays are bound to spatial search to find PUs, while the proposed method bypasses spatial search by estimating PUs locations via integrating PIMs from differnt sensors mounted at different locations around the area of interest. This means a reduction in the required time for PUs localization. Therefore, the proposed technique significantly reduces the number of required antennas, the complexity of the receiver and the required time for localization compared to the network that exploits the DOA technique. On the other hand, the number of detectable PUs using the proposed technique is limited up to the bound of (13), while the network that utilizes antenna arrays is capable of detecting more PUs and the only limiting parameter on the number of PUs is the resolution of localization.

\section{CONCLUSION}

In this work, we proposed a localization framework based on mounting two antennas on each sensor of a sensor net- 
work. The antennas are placed multiple wavelength apart. we measure phase differences at each pair of antennas provoked by signal emission of PUs using a tensor decomposition technique. We showed that using PIMs, highly accurate location estimation of PUs can be obtained. We showed that the proposed technique can perform similar to a sensor network that utilizes very large ULA antennas which is more complex than our proposed configuration in terms of implementation. We discussed that the proposed technique can greatly reduces the complexity, the cost and the time required for a sensor network to localize multiple PUs in an area of interest in comparison to a sensor network that utilizes electronically steerable antennas.

\section{REFERENCES}

[1] Richard G Wiley, ELINT: The interception and analysis of radar signals, Artech House, 2006.

[2] Abdelmohsen Ali and Walaa Hamouda, "Advances on spectrum sensing for cognitive radio networks: Theory and applications," IEEE communications surveys \& tutorials, vol. 19, no. 2, pp. 1277-1304, 2016.

[3] Juan Andrés Bazerque and Georgios B Giannakis, "Distributed spectrum sensing for cognitive radio networks by exploiting sparsity," IEEE Transactions on Signal Processing, vol. 58, no. 3, pp. 1847-1862, 2009.

[4] Mohsen Joneidi, Hassan Yazdani, Azadeh Vosoughi, and Nazanin Rahnavard, "Source localization and tracking for dynamic radio cartography using directional antennas," in 2019 16th Annual IEEE International Conference on Sensing, Communication, and Networking (SECON). IEEE, 2019, pp. 1-9.

[5] Ehsan Taghavi, Ratnasingham Tharmarasa, Thia Kirubarajan, and Mike Mcdonald, "Multisensor-multitarget bearing-only sensor registration," IEEE Transactions on Aerospace and Electronic Systems, vol. 52, no. 4, pp. 1654-1666, 2016.

[6] MM Khalili, F Hejazi, Y Norouzi, and MM Nayebi, "Secant method for bearing-only tracking problem," in 2013 14th International Radar Symposium (IRS). IEEE, 2013, vol. 1, pp. 393-398.

[7] Nur Aisyah Azmi, Shafiqa Samsul, Yoshihide Yamada, Mohd Fitri Mohd Yakub, Mohd Ismifaizul Mohd Ismail, and Rudzidatul Akmam Dziyauddin, "A survey of localization using rssi and tdoa techniques in wireless sensor network: System architecture," in 2018 2nd International Conference on Telematics and Future Generation Networks (TAFGEN). IEEE, 2018, pp. 131-136.

[8] KC Ho, Xiaoning Lu, and La-or Kovavisaruch, "Source localization using tdoa and fdoa measurements in the presence of receiver location errors: Analysis and solution," IEEE Transactions on Signal Processing, vol. 55, no. 2, pp. 684-696, 2007.

[9] Hyeondeog Seo, Hyowon Kim, Jeongwan Kang, Incheol Jeong, Woogeun Ahn, and Sunwoo Kim, "3d moving target tracking with measurement fusion of tdoa/fdoa/aoa," ICT Express, vol. 5, no. 2, pp. 115-119, 2019.

[10] Nil Garcia, Henk Wymeersch, Erik G Larsson, Alexander M Haimovich, and Martial Coulon, "Direct localization for massive mimo," IEEE Transactions on Signal Processing, vol. 65, no. 10, pp. 2475-2487, 2017.

[11] Ying-Wah Wu, Stephen Rhodes, and Edgar H Satorius, "Direction of arrival estimation via extended phase interferometry," IEEE transactions on aerospace and electronic systems, vol. 31, no. 1, pp. 375-381, 1995.

[12] Farzam Hejazikookamari, Yaser Norouzi, Elham Sadat Kashani, and Mohammad Mahdi Nayebi, "A novel method to detect and localize lpi radars," IEEE Transactions on Aerospace and Electronic Systems, vol. 55, no. 5, pp. 2327-2336, 2018.

[13] Farzam Hejazi Kookamari, Yaser Norouzi, and Mohammad Mahdi Nayebi, "Using a moving aerial platform to detect and localise a low probability of intercept radar," IET Radar, Sonar \& Navigation, vol. 11, no. 7, pp. 1062-1069, 2017.

[14] Stephen Searle, "Disambiguation of interferometric doa estimates in vehicular passive radar," IET Radar, Sonar \& Navigation, vol. 12, no. 1, pp. 64-73, 2017.

[15] Sajjad Mollai and Forouhar Farzaneh, "Compact cross form antenna arrays intended for wideband two dimensional interferometric direction finding including the channel phase tracking error," AEU-International Journal of Electronics and Communications, vol. 83, pp. 558-565, 2018.
[16] Nicholas D Sidiropoulos, Georgios B Giannakis, and Rasmus Bro, "Blind parafac receivers for ds-cdma systems," IEEE Transactions on Signal Processing, vol. 48, no. 3, pp. 810-823, 2000.

[17] Michele Nazareth da Costa, Gérard Favier, and João Marcos T Romano, "Tensor modelling of mimo communication systems with performance analysis and kronecker receivers," Signal Processing, vol. 145, pp. 304$316,2018$.

[18] Mikael Sørensen, Frederik Van Eeghem, and Lieven De Lathauwer, "Blind multichannel deconvolution and convolutive extensions of canonical polyadic and block term decompositions," IEEE Transactions on Signal Processing, vol. 65, no. 15, pp. 4132-4145, 2017.

[19] Giancarlo Pastor, "A low-rank tensor model for imputation of missing vehicular traffic volume," IEEE Transactions on Vehicular Technology, vol. 67, no. 9, pp. 8934-8938, 2018.

[20] Cheng Qian, Xiao Fu, Nicholas D Sidiropoulos, and Ye Yang, "Tensorbased channel estimation for dual-polarized massive mimo systems," IEEE Transactions on Signal Processing, vol. 66, no. 24, pp. 63906403, 2018

[21] Mojtaba Taherisadr, Mohsen Joneidi, and Nazanin Rahnavard, "EEG signal dimensionality reduction and classification using tensor decomposition and deep convolutional neural networks," in 2019 IEEE 29th International Workshop on Machine Learning for Signal Processing $(M L S P)$. IEEE, 2019, pp. 1-6.

[22] Xiao Fu, Nicholas D Sidiropoulos, and Wing-Kin Ma, "Tensor-based power spectra separation and emitter localization for cognitive radio," in 2014 IEEE 8th Sensor Array and Multichannel Signal Processing Workshop (SAM). IEEE, 2014, pp. 421-424.

[23] X. Fu, N. D. Sidiropoulos, J. H. Tranter, and W. K. Ma, "A factor analysis framework for power spectra separation and multiple emitter localization," IEEE Transactions on Signal Processing, vol. 63, no. 24 pp. 6581-6594, Dec 2015.

[24] Michael M Goodwin and Gary W Elko, "Constant beamwidth beamforming," in 1993 IEEE International Conference on Acoustics, Speech, and Signal Processing. IEEE, 1993, vol. 1, pp. 169-172.

[25] Xiao Fu, Nicholas D Sidiropoulos, and Wing-Kin Ma, "Power spectra separation via structured matrix factorization," IEEE Transactions on Signal Processing, vol. 64, no. 17, pp. 4592-4605, 2016.

[26] Tamara G Kolda and Brett W Bader, "Tensor decompositions and applications," SIAM review, vol. 51, no. 3, pp. 455-500, 2009.

[27] M. Salimitari, M. Joneidi, and M. Chatterjee, "Ai-enabled blockchain: An outlier-aware consensus protocol for blockchain-based iot networks," in 2019 IEEE Global Communications Conference (GLOBECOM), Dec.

[28] Nicholas D Sidiropoulos, Lieven De Lathauwer, Xiao Fu, Kejun Huang, Evangelos E Papalexakis, and Christos Faloutsos, "Tensor decomposition for signal processing and machine learning," IEEE Transactions on Signal Processing, vol. 65, no. 13, pp. 3551-3582, 2017.

[29] Mathias Lohne, "The computational complexity of the fast fourier transform,” Tech. Rep., Tech. Rep., 2017.[Online]. Available: https://folk.

[30] Casey Battaglino, Grey Ballard, and Tamara G Kolda, "A practical randomized cp tensor decomposition," SIAM Journal on Matrix Analysis and Applications, vol. 39, no. 2, pp. 876-901, 2018.

[31] Alfonso Farina, "Target tracking with bearings-only measurements," Signal processing, vol. 78, no. 1, pp. 61-78, 1999.

[32] F Hejazi, Yaser Norouzi, and Mohammad Mehdi Nayebi, "Lower bound of error in aoa based passive source localization using single moving platform," in East-West Design \& Test Symposium (EWDTS 2013). IEEE, 2013, pp. 1-4.

[33] Federico Penna and Danijela Cabric, "Bounds and tradeoffs for cooperative doa-only localization of primary users," in 2011 IEEE Global Telecommunications Conference-GLOBECOM 2011. IEEE, 2011.

[34] Robert J Mailloux, Phased array antenna handbook, Artech house, 2017.

[35] F Hejazi, MM Khalili, Y Norouzi, and MM Nayebi, "A new pseudolinear solution to bearing-only tracking," in 2013 IEEE Radar Conference (RadarCon13). IEEE, 2013, pp. 1-4.

[36] Shajahan Kutty and Debarati Sen, "Beamforming for millimeter wave communications: An inclusive survey," IEEE Communications Surveys \& Tutorials, vol. 18, no. 2, pp. 949-973, 2015. 